\section{Nonlinear Robust Controller Design for Multi-Robot Systems with Unknown Payloads}

\author{
Y. D. Song \\ J. N. Anderson ${ }^{\star}$ \\ A. Homaifar \\ H. Y. Lai \\ NASA Center for Aerospace Research \\ North Carolina A\&T State University, Greensboro, NC 27411 \\ * Center for Manufacturing Research \\ Tennessee Technological University, Cookeville, TN 38505
}

\begin{abstract}
This work is concerned with the control problem of a multi-robot system handling a payload with unknown mass properties. Force constraints at the grasp points are considered. Robust control schemes are proposed that cope with the model uncertainty and achieve asymptotic path tracking. To deal with the force constraints, a strategy for optimally sharing the task is suggested. This strategy basically consists of two steps. The first detects the robots that need help and the second arranges that help. It is shown that the overall system is not only robust to uncertain payload parameters, but also satisfies the force constraints.
\end{abstract}

Keywords: Multi-robot systems, unknown payloads, robust control, adaptive control, force constraints.

\section{INTRODUCTION}

The range of tasks that require anthropomorphic manipulation motivates the use of multi-robot systems. In particular, tasks that require manipulation of a single heavy load or a cumbersome object could exceed the force and work envelope limits of a single arm. One of the major issues involved in a multi-robot system is to coordinate all the robots so that they perform a given task in a cooperative manner. More specifically, the control problem includes dynamic behavior modeling, trajectory tracking control, internal force control and task distribution among the robots.

Although still in its early stages of development, there has been significant progress in this area, and a great deal of work has been reported in recent years. In the area of modeling, the work by Luh and Zheng [14] is among the earliest research dealing with the kinematic and dynamic constraints imposed on such a system. This was also studied for a two-arm system by Suh and Shin [28].
The dynamics model of a multi-robot system is required for the development of control algorithms. As has been reported in the literature, the dynamics model of a multi-robot system exhibits its own inherent properties. Hayati [9] investigated a dynamic model for a closed-chain system. This issue, together with the control problem, has also been studied by Tarn, Bejczy and Yun [29], Furuta, et al [8], Özgüner, Yurkovich and AlAbbass [18], Yun [35] and Li, Hsu and Sastry [13].

The work by Cole, Hause and Sastry [6] considered the case of a multifingered hand in rolling contact with an object and both the kinematics and control issues were investigated. Alford and Belyeu [2] studied a two-arm system and proposed a leader-follower control strategy, which was generalized to a multi-arm system by Arimoto, Miyazaki and Kawamura [4]. A position and force control scheme for a multi-robot system was proposed by Nakamura, Nagai and Yoshikawa [16].

Issues related to force distribution in closed kinematic chains were discussed by Orin and $\mathrm{Oh}[17]$. Alberts and Soloway [1] suggested a control law which distributes force among the manipulators by using a weighting function. In the work by Salisbury and Craig [21], Yoshikawa and Nagai [34] and Nakamura, Nagai and Yoshikawa [16], the force exerted on the object is distributed based on an object-related criterion. Zheng and Luh [36] developed load distribution schemes for two-manipulator systems which were based on minimizing either the system energy or the force imparted to the object. In those schemes, the manipulator's dynamics are explicitly considered. Anderson and Pittelkau [3] proposed a load sharing force controller that apportions control forces between two manipulators. An adaptive algorithm that uses a joint torque-based criterion to determine the optimum load sharing was suggested in that work.

By considering the effect of loading on the dynam- 
ics constraints, Walker, Marcus and Freeman [32] presented an approach for load distribution. The reaction forces created by a given arm's input loading was considered in Unseren and Koivo [30]. Carignan and Akin [5] derived the torques for two planar cooperating arms. A coordinated control law for a multimanipulator system performing parts-matching tasks was proposed by Hsu [10] and a decentralized structure for the control strategy was suggested. In a recent work by Walker, Freeman and Marcus [31], the motion and internal loads induced on an object grasped by two or more robotic manipulators were analyzed and the load distribution problem was formulated by using the nonsqueezing pseudoinverse.

In the context of controller design, two approaches are generally used. In the first, the problem is simplified by implicitly assuming that the payload information is known exactly and can be precisely modelled. In the second approach uncertain payload effects are compensated by the use of a wrist force sensor (see references $[3],[9],[19]$ and [29]). In general precise information about the payload is not available for many applications, and the force sensor method depends heavily on the precision of the force measurement, as has been shown in [3]. These facts motivate the study of adaptive control schemes for multi-robot systems.

Mo and Bayoumi [15] proposed an adaptive control method for a multi-arm robotic system by using a method similar to that in Craig, Hsu and Sastry [7]. Walker, Kim and Dionise [33] reported an adaptive control strategy that accounts for payload effects. Hu and Goldenberg [11] also investigated the case of uncertain parameters in a multi-arm system. However, in most of these strategies the force constraint problem is not considered.

In a recent work by Song and Anderson [23], a new adaptive control law with a colleague-like strategy for task distribution was reported in which the force constraints are explicitly considered. As an extension of and a complement to that work, this paper is devoted to the robust controller design for multi-robot systems with both unknown payload dynamics and force constraints. First, following a modeling procedure similar to that in [6], a combined dynamic model which accounts for payload effects is derived. This model is slightly different in structure from the one obtained in [6] as a result of considering the forces and the moments exerted on the object. New robust control algorithms that explicitly deal with unknown payload parameters are developed. By using the generalized energy accumulation method, it is shown that the control strategy guarantees asymptotically stable path tracking of the payload's mass center. A strategy for task distribution is suggested which considers the dual contributions of the control torques. That is, it not only produces the control torques required for path tracking, but also satisfies the force constriants, a necessary condition for fine manipulation of the workpiece.

The main uncertainty in practice is due to the payload, not the robots themselves. Thus the attention in this paper is focused on the uncertain payload - the most significant effect on system performance (Leahy [12]). However, the technique presented herein can be readily extended to the case where uncertain parameters exist in both the robot and payload models.

The remainder of this paper is organized as follows. Section 2 presents a derivation of the combined multirobot/payload dynamics model based on the fundamental equations presented in the first part of the section. The structural properties of this model are investigated, and are utilized in Section 3 to develop robust control strategies. Tracking stability is analyzed in Section 4 , and a new robust control which does not rely on norm bounds is proposed in Section 5. Task distribution among the robots is discussed in Section 6 and a strategy for optimal sharing of the task is proposed. The application of the strategy is illustrated in Section 7 by means of a three-robot example. Comments and conclusions are given in Section 8.

\section{MODELING}

A dynamic model for a multi-robot system handling a workpiece was developed in [23]. However, due to limited space, many important details were omitted. For completeness, the modeling process of [23] is expanded in the following.

\subsection{Kinematics}

The multi-robot system illustrated in Figure 1 represents several robots handling a common payload. For simplicity, it is assumed that each robot has six degreesof-freedom. The first joint of each robot is attached to a fixed base and the $i^{\text {th }}$ robot is grasping the payload at the contact point $C_{i}$. Rigid grasping is assumed such that there is no relative motion at the contact points and there is perfect force transmission between the robots and the payload. Also each contact point is fixed and has a known location on the payload. Each robot applies a force $f_{c_{1}}$ and a moment $n_{c_{\text {, }}}$ through the contact point $C_{i}$ to the payload. There are totally $d(d \geq 2)$ robots handling the payload, and the payload lies within the combined loading capacity of the robots.

The Cartesian coordinate frames shown in Figure 2 are defined as follows:

$\left\{F_{f}\right\}$ is the inertial reference frame.

$\left\{F_{p}\right\}$ is the frame fixed at the payload's center-ofmass. 
Figure 1: A Multi-Robot System

$\left\{F_{b_{i}}\right\}$ is the frame fixed to the base of the $i^{\text {th }}$ robot. $\left\{F_{c_{i}}\right\}$ is the frame attached to the $i^{\text {th }}$ end-effector at the contact point $C_{i}$.

According to Chasle's theorem [22] from elementary mechanics, the motion of a rigid body in world space can decomposed into a translation plus a rotation. This is referred to as a rigid motion by Spong and Vidyasagar [27]. Six parameters are required to describe the position and orientation of the rigid body.

At the contact point $C_{i}$ (see Figure 2),

$$
x_{c_{i}}=x_{0}+R_{p}^{f} c_{i}+d_{i}
$$

and

$$
\omega_{c_{i}}=\omega_{0}
$$

where

$d_{i}$ is the vector that locates the origin of $\left\{F_{b_{i}}\right\}$ relative to $\left\{F_{\rho}\right\}$,

$c_{i}$ is the vector that locates the origin of $\left\{F_{c_{i}}\right\}$ relative to $\left\{F_{\mathrm{p}}\right\}$,

$x_{0}=\left[x_{0 x}, x_{0 y}, x_{0 z}\right]^{T}$ is a vector in $\left\{F_{f}\right\}$ locating the mass-center of the payload,

$\omega_{0}=\left[\omega_{0 y}, \omega_{0 p}, \omega_{0 r}\right]^{T}$ is the angular velocity vector of $\left\{F_{p}\right\}$ in terms of the yaw, pitch and roll rates,

$\omega_{c_{i}}$ is the angular velocity of $\left\{F_{c_{i}}\right\}$, and

$R_{p}^{j}$ is the rotation matrix which maps $c_{i}$, measured in frame $\left\{F_{p}\right\}$, to the reference frame $\left\{F_{f}\right\}$.

In much of the literature, it is implicitly assumed that $R_{p}^{f}=E_{3} \in R^{3 \times 3}$, a unit matrix. This is true if
Figure 2: Coordinate Frames

the frames $\left\{F_{p}\right\}$ and $\left\{F_{f}\right\}$ have the same orientation. However, since $R_{p}^{j}$ depends on $\phi_{0}=\left[\phi_{0 y}, \phi_{0 p}, \phi_{0 r}\right]^{T}$, the yaw, pitch and roll angles of frame $\left\{F_{p}\right\}$, respectively, it is generally not true that $R_{p}^{J}=E_{3}$ as the payload undergoes rotational motion. Hence, in the following, a time varying $R_{p}^{J}$ is considered. To simplify notation, $R$ is used to denote $R_{p}^{f}$ in the following.

As can be verified, the matrix $R$ has the property [27]

$$
\dot{R}=\omega_{0} \times R
$$

where

$$
\omega_{0} \times=\left[\begin{array}{ccc}
0 & -\omega_{0 p} & \omega_{0 r} \\
\omega_{0 p} & 0 & -\omega_{0 y} \\
-\omega_{0 r} & \omega_{0 y} & 0
\end{array}\right] .
$$

Differentiating (1) and noting that $d_{i}$ and $c_{i}$ are constant yields

$$
\begin{aligned}
\dot{x}_{c_{i}} & =\dot{x}_{0}+\dot{R} c_{i} \\
& =\dot{x}_{0}+\omega_{0} \times R c_{i} .
\end{aligned}
$$

Since $\omega_{0} \times R c_{i}=-\left(R c_{i}\right) \times \omega_{0},(2)$ and (4) can be written as

$$
\begin{aligned}
{\left[\begin{array}{l}
\dot{x}_{c_{1}} \\
\omega_{c_{i}}
\end{array}\right] } & =\left[\begin{array}{cc}
E_{3} & -\left(R c_{i}\right) \times \\
0 & E_{3}
\end{array}\right]\left[\begin{array}{l}
\dot{x}_{0} \\
\omega_{0}
\end{array}\right] \\
& \triangleq S_{i}\left[\begin{array}{l}
\dot{x}_{0} \\
\omega_{0}
\end{array}\right]
\end{aligned}
$$


where

$$
S_{i}=\left[\begin{array}{cc}
E_{3} & -\left(R c_{i}\right) \times \\
0 & E_{3}
\end{array}\right] .
$$

In the task space, the position and orientation of the $i^{\text {th }}$ end-effector can be represented as

$$
\begin{aligned}
P_{i} & =\left[x_{c_{i}}^{T}, \phi_{c_{i}}^{T}\right]^{T} \\
& =\left[x_{c i x}, x_{c i y}, x_{c i x}, \phi_{c i y}, \phi_{c i p}, \phi_{c i r}\right]^{T}
\end{aligned}
$$

where $\phi_{c i}$ is composed of the yaw, pitch and roll angles representing the orientation of the $i^{\text {th }}$ end-effector with respect to $\left\{F_{f}\right\}$. In joint space $P_{i}$ can be expressed as

$$
P_{i}=P_{i}\left(q_{i}\right),
$$

where $q_{i} \in R^{6}$ is the generalized joint displacement of the $i^{\text {th }}$ robot and $P_{i}():. R^{6} \rightarrow R^{6}$ represents its forward kinematics. Therefore,

$$
\begin{aligned}
\dot{P}_{i} & =\left[\dot{x}_{c_{i}}^{T}, \dot{\phi}_{c i}^{T}\right]^{T} \\
& =\frac{\partial P_{i}\left(q_{i}\right)}{\partial q_{i}} \dot{q}_{i} .
\end{aligned}
$$

Since

$$
\omega_{c_{i}}=T_{c_{i}}\left(\phi_{c i y}, \phi_{c i p}, \phi_{c i r}\right)\left[\begin{array}{l}
\dot{\phi}_{c i y} \\
\dot{\phi}_{c i p} \\
\dot{\phi}_{c i r}
\end{array}\right],
$$

where

$$
T_{c_{i}}(.)=\left[\begin{array}{ccc}
0 & -\sin \phi_{c i y} & \cos \phi_{c i p} \cos \phi_{c i y} \\
0 & \cos \phi_{c i y} & \cos \phi_{c i p} \sin \phi_{c i y} \\
1 & 0 & -\sin \phi_{c i p}
\end{array}\right],
$$

then using (5), (9) and (10) yields

$$
S_{i}\left[\begin{array}{c}
\dot{x}_{0} \\
\omega_{0}
\end{array}\right]=J_{i} \dot{q}_{i}
$$

where

$$
J_{i}=\left[\begin{array}{cc}
E_{3} & 0 \\
0 & T_{c_{i}}\left(\phi_{\text {eiy }}, \phi_{\text {cip }}, \phi_{\text {eir }}\right)
\end{array}\right] \frac{\partial P_{i}\left(q_{i}\right)}{\partial q_{i}}
$$

is the generalized Jacobian matrix of robot $i$. It is assumed in the following that each robot works in a nonsingular region. Thus the inverse of $J_{i}$ exists.

Considering all the robots that act on the payload, (12) can be expressed compactly as

$$
\mathbf{J} \dot{\mathbf{q}}=\mathbf{S}\left[\begin{array}{c}
\dot{x}_{0} \\
\omega_{0}
\end{array}\right]
$$

where

$$
\begin{aligned}
& \mathbf{J} \triangleq \operatorname{blockdiag}\left\{J_{1}, J_{2}, \ldots, J_{d}\right\} \in R^{6 d \times 6 d} \\
& \mathbf{S} \triangleq\left[S_{1}^{T} S_{2}^{T} \ldots S_{d}^{T}\right]^{T} \in R^{6 d \times 6}
\end{aligned}
$$

and

$$
\mathrm{q} \triangleq\left[q_{1}^{T} q_{2}^{T} \ldots q_{d}^{T}\right]^{T} \in R^{6 d} .
$$

Since the forces $\left\{f_{c 1}, f_{c 2}, \ldots, f_{c d}\right\}$ and moments $\left\{n_{c 1}, n_{c 2}, \ldots, n_{c d}\right\}$ act on the load, the equivalent force and moment applied at the mass-center are

$$
f_{0}=f_{c_{1}}+f_{c_{3}}+\ldots+f_{c_{d}}
$$

and

$$
\begin{aligned}
n_{0}= & n_{c_{1}}+n_{c_{2}}+\ldots+n_{c_{d}}+\left(R c_{1}\right) \times f_{c_{1}} \\
& +\left(R c_{2}\right) \times f_{c_{3}}+\ldots+\left(R c_{d}\right) \times f_{c_{d}}
\end{aligned}
$$

respectively. In matrix form, (15) and (16) become

$$
\begin{aligned}
{\left[\begin{array}{c}
f_{0} \\
n_{0}
\end{array}\right]=} & {\left[\begin{array}{cc}
E_{3} & 0 \\
\left(R c_{1}\right) \times & E_{3}
\end{array}\right] F_{1}+\left[\begin{array}{cc}
E_{3} & 0 \\
\left(R c_{2}\right) \times & E_{3}
\end{array}\right] F_{2} } \\
& +\ldots+\left[\begin{array}{cc}
E_{3} & 0 \\
\left(R c_{d}\right) \times & E_{3}
\end{array}\right] F_{d} \\
= & W_{1} F_{1}+W_{2} F_{2}+\ldots+W_{d} F_{d} \\
\triangleq & \mathrm{WF}
\end{aligned}
$$

where

$$
\begin{aligned}
& F_{i} \triangleq\left[\begin{array}{l}
f_{c_{i}} \\
n_{c_{i}}
\end{array}\right], \quad W_{i} \triangleq\left[\begin{array}{cc}
E_{3} & 0 \\
\left(R c_{i}\right) \times & E_{3}
\end{array}\right] \\
& \mathbf{W} \triangleq\left[W_{1} W_{2} \ldots W_{d}\right] \in R^{6 \times 6 d}
\end{aligned}
$$

and

$$
\mathbf{F} \triangleq\left[\begin{array}{lll}
F_{1}^{T} & F_{2}^{T} \ldots F_{d}^{T}
\end{array}\right]^{T} \in R^{6 d} .
$$

Concerning the matrices $\mathbf{W}$ and $\mathbf{S}$, the following properties hold.

\section{Property 2.1}

(1) $\mathbf{S}$ and $\mathbf{W}$ are full $\operatorname{rank}$, i.e., $\operatorname{rank}(\mathbf{W})=\operatorname{rank}(\mathbf{S})=$ 6.

(2) Both $S_{i}$ and $W_{i}$ are nonsingular, $i=1,2, \ldots, d$.

(3) $\mathbf{S}^{T}=\mathrm{W}$

The proof is similar to that given by Song and Anderson [24].

\subsection{Motion Equation}

Suppose that the mass and the inertia of the payload is $m$ and $I_{0}$, respectively. With the action of $f_{0}$ and $n_{0}$, the payload undergoes a rigid motion in the world space as described by

$$
f_{0}=m \ddot{x}_{0}+m g
$$

and

$$
n_{0}=R I_{0} R^{T} \dot{\omega}_{0}+\omega_{0} \times R I_{0} R^{T} \omega_{0},
$$

where $g \in R^{3}$ is the gravity vector. In view of (17)(19),

$$
\mathbf{W F}=\mathcal{D}_{1}\left[\begin{array}{c}
\ddot{x}_{0} \\
\dot{\omega}_{0}
\end{array}\right]+\mathcal{D}_{2}\left[\begin{array}{l}
\dot{x}_{0} \\
\omega_{0}
\end{array}\right]+\mathcal{D}_{3}
$$


where

$$
\begin{aligned}
& \mathcal{D}_{1}=\left[\begin{array}{cc}
m E_{3} & 0 \\
0 & R I_{0} R^{T}
\end{array}\right] \\
& \mathcal{D}_{2}=\left[\begin{array}{cc}
0 & 0 \\
0 & \omega_{0} \times R I_{0} R^{T}
\end{array}\right]
\end{aligned}
$$

and

$$
\mathcal{D}_{3}=\left[\begin{array}{c}
m g \\
0
\end{array}\right] \text {. }
$$

The reactive forces and moments of the payload appearing at the $i^{\text {th }}$ end-effector are represented as $F_{\text {react }}$. Under the assumption of rigid grasping at the contact point,

$$
F_{\text {react, }}=-F_{i},
$$

which causes the joint reaction torques

$$
\begin{aligned}
\tau_{F_{\text {reecti }}} & =J_{i}^{T}(q) F_{\text {react }_{i}} \\
& =-J_{i}^{T}\left(g_{i}\right) F_{i} .
\end{aligned}
$$

So for each robot it follows that

$$
\begin{aligned}
H_{i} \ddot{q}_{i}+C_{i} \dot{q}_{i} & +G_{i}=\tau_{i}+\tau_{F_{\text {recct }}} \text { number } \\
& =\tau_{i}-J_{i}^{T} F_{i}, \quad i=1,2, \ldots, d
\end{aligned}
$$

where, for the $i^{\text {th }}$ robot, $H_{i}$ is the inertia matrix, $C_{i} \dot{q}_{i}$ is the vector of centrifugal and Corolis forces, $G_{i}$ is the vector of gravitational forces and $\tau_{i}$ is the vector of control torques. Defining

$$
\begin{aligned}
& \mathbf{H} \triangleq \text { block } \operatorname{diag}\left\{H_{1}, H_{2}, \ldots H_{d}\right\} \in R^{6 d \times 6 d}, \\
& \mathbf{C} \triangleq \text { block } \operatorname{diag}\left\{C_{1}, C_{2}, \ldots C_{d}\right\} \in R^{6 d \times 6 d}, \\
& \mathbf{G} \triangleq\left[G_{1}^{T} G_{1}^{T} \ldots G_{d}^{T}\right]^{T} \in R^{6 d},
\end{aligned}
$$

and

$$
\mathbf{T} \triangleq\left[\begin{array}{lll}
\tau_{1}^{T} & \tau_{2}^{T} \ldots \tau_{d}^{T}
\end{array}\right]^{T} \in R^{6 d},
$$

the combined form of $(25)$ is

$$
\mathbf{T}=\mathbf{H} \ddot{\mathbf{q}}+\mathbf{C} \dot{\mathbf{q}}+\mathbf{G}+\mathbf{J}^{T} \mathbf{F} .
$$

This model can easily be transformed to the task space. From (14)

$$
\dot{\mathbf{q}}=\mathbf{J}^{-1} \mathbf{S}\left[\begin{array}{c}
\dot{x}_{0} \\
\omega_{0}
\end{array}\right]
$$

Noting that

$$
\begin{aligned}
\omega_{0} & =T_{0}\left(\phi_{0 y}, \phi_{0 p}, \phi_{0 r}\right)\left[\begin{array}{l}
\dot{\phi}_{0 y} \\
\dot{\phi}_{0 p} \\
\dot{\phi}_{0 r}
\end{array}\right] \\
& \triangleq T_{0} \dot{\phi}_{0},
\end{aligned}
$$

where

$$
T_{0}(.)=\left[\begin{array}{ccc}
0 & -\sin \phi_{0 y} & \cos \phi_{0 p} \cos \phi_{0 y} \\
0 & \cos \phi_{0 y} & \cos \phi_{0 p} \sin \phi_{0 y} \\
1 & 0 & -\sin \phi_{0 p}
\end{array}\right]
$$

it follows that

$$
\begin{aligned}
{\left[\begin{array}{l}
\dot{x}_{0} \\
\omega_{0}
\end{array}\right] } & =\left[\begin{array}{cc}
E_{3} & 0 \\
0 & T_{0}
\end{array}\right]\left[\begin{array}{l}
\dot{x}_{0} \\
\dot{\phi}_{0}
\end{array}\right] \\
& \triangleq A \dot{X}_{0}
\end{aligned}
$$

where

$$
A=\left[\begin{array}{cc}
E_{3} & 0 \\
0 & T_{0}
\end{array}\right] \in R^{6 \times 6}
$$

and

$$
X_{0}=\left[\begin{array}{l}
x_{0} \\
\phi_{0}
\end{array}\right] .
$$

Notice that $\operatorname{det}\left(T_{0}\right)=-\cos \phi_{0 p}$. For small changes in the payload's orientation $\left(\left|\phi_{0 p}\right|<\frac{\pi}{2}\right)$, the matrix $A$ is invertible. Now by (27) and (29),

$$
\dot{\mathbf{q}}=\mathbf{J}^{-1} \mathbf{S} A \dot{X}_{0}
$$

such that

$$
\ddot{\mathbf{q}}=\mathbf{J}^{-1} \mathbf{S} A \ddot{X}_{0}+\frac{d}{d t}\left(\mathrm{~J}^{-1} \mathbf{S} A\right) \dot{X}_{0} .
$$

In view of $(20)-(22),(26)$ and $(29)-(31)$, the combined dynamics of the multi-robot system through the payload may be written as

$$
\begin{aligned}
\mathbf{W J}^{-T} \mathbf{T}= & \left(\mathbf{W J}^{-T} \mathbf{H J}^{-1} \mathbf{S} A+\mathcal{D}_{1} A\right) \ddot{X}_{0} \\
& +\left(\mathbf{W J} \mathbf{J}^{-T} \mathbf{H} \frac{d}{d t}\left(\mathbf{J}^{-1} \mathbf{S} A\right)\right. \\
& \left.+\mathbf{W J}^{-T} \mathbf{C J}^{-1} \mathbf{S} A+\mathcal{D}_{1} \dot{A}+\mathcal{D}_{2} A\right) \dot{X}_{0} \\
& +\mathbf{W J}^{-T} \mathbf{G}+\mathcal{D}_{3}
\end{aligned}
$$

It turns out that the combined model is somewhat "irregular" in that the well-known properties applicable to general robot dynamics do not necessarily hold. That is;

1. the generalized inertia matrix $\mathrm{WJ}^{-T} \mathrm{HJ}^{-1} \mathrm{~S} A+$ $\mathcal{D}_{1} A$ is not symmetric, and

2. the property of skew-symmetry is not valid.

\section{Remark 2.1}

The fact that the generalized inertia matrix is not symmetric positive definite makes it difficult to directly use the Lyapunov method to verify stability. The passivity principle also cannot be directly 
applied because the property of skew-symmetry does not hold. In the next section, a simple transformation is applied to "regularize" the combined model.

\section{ROBUST CONTROL METHODS}

\subsection{Control Objective}

As previously noted, it is generally difficult to know the payload's characteristics precisely. For this reason, it is assumed that the mass $m$ and the inertia matrix $I_{0}$ of the payload are unknown. The desired path is given in terms of position and orientation as

$$
\begin{aligned}
& X_{0}^{d}=\left[\begin{array}{l}
x_{0}^{d} \\
\phi_{0}^{d}
\end{array}\right], \\
& \dot{X}_{0}^{d}=\left[\begin{array}{l}
\dot{x}_{0}^{d} \\
\dot{\phi}_{0}^{d}
\end{array}\right],
\end{aligned}
$$

and

$$
\ddot{X}_{0}^{d}=\left[\begin{array}{c}
\ddot{x}_{0}^{d} \\
\ddot{\phi}_{0}^{d}
\end{array}\right] \text {. }
$$

The tracking error is expressed as

$$
\begin{aligned}
\varepsilon & =X_{0}-X_{0}^{d} \\
& =\left[\begin{array}{l}
x_{0}-x_{0}^{d} \\
\phi_{0}-\phi_{0}^{d}
\end{array}\right] \triangleq\left[\begin{array}{l}
\varepsilon_{1} \\
\varepsilon_{2}
\end{array}\right] .
\end{aligned}
$$

The motion control problem is stated as:

Design the control torque $\mathbf{T}$ such that the actual path of the payload's mass center $\left(X_{0}\right)$ asymptotically tracks the desired path $\left(X_{0}^{d}\right)$ in the face of unknown payload parameters.

\subsection{Model Transformation}

Due to the "irregularity" of the model (32), direct solution to this problem is complicated. In the following, a simple transformation is introduced. By observing the structure of the combined model (32), it is seen that if both sides are premultiplied by $A^{T}$, the transformed generalized inertia matrix is symmetric positive definite. Moreover, this mutiplication also leads to the property of skew-symmetry. After the transformation, (32) becomes

$$
\left[\mathcal{H}_{0}+\mathcal{H}_{p}\right] \ddot{X}_{0}+\left[\mathcal{C}_{0}+\mathcal{C}_{p}\right] \dot{X}_{0}+\left[\mathcal{G}_{0}+\mathcal{G}_{p}\right]=\mathcal{F},
$$

where

$$
\begin{aligned}
\mathcal{F} & \triangleq A^{T} \mathbf{W J}^{-T} \mathbf{T} \\
\mathcal{H}_{0} & \triangleq A^{T} \mathbf{W J} \mathbf{J}^{-T} \mathbf{H J}^{-1} \mathbf{S} A \\
\mathcal{H}_{p} & \triangleq A^{T} \mathcal{D}_{1} A \\
& =\left[\begin{array}{cc}
m E_{3} & 0 \\
0 & T_{0}^{T} R I_{0} R^{T} T_{0}
\end{array}\right],
\end{aligned}
$$

$$
\begin{aligned}
\mathcal{C}_{0}= & A^{T} \mathbf{W J}^{-T} \mathbf{H}\left(\mathbf{J}^{-1} \mathbf{S} A+\mathbf{J}^{-1} \dot{\mathbf{S}} A+\mathbf{J}^{-1} \mathbf{S} \dot{A}\right) \\
& +A^{T} \mathbf{W J}^{-T} \mathbf{C J}^{-1} \mathbf{S} A, \\
\mathcal{C}_{p} \triangleq & A^{T} \mathcal{D}_{1} \dot{A}+A^{T} \mathcal{D}_{2} A \\
= & {\left[\begin{array}{lc}
0 & 0 \\
0 & T_{0}^{T} \omega_{0} \times R I_{0} R^{T} T_{0}+T_{0}^{T} R I_{0} R^{T} T_{0}
\end{array}\right], }
\end{aligned}
$$

$$
\mathcal{G}_{0} \triangleq A^{T} \mathbf{W J}^{-T} \mathbf{G}
$$

and

$$
\mathcal{G}_{\mathrm{p}} \triangleq A^{T} \mathcal{D}_{3}=\left[\begin{array}{c}
m g \\
0
\end{array}\right] \text {. }
$$

For this model the following properties hold.

\section{Theorem 3.1}

The transformed model (33) is regular. That is;

(1) $\mathcal{H}_{0}+\mathcal{H}_{p}$ is symmetric positive definite, and

(2) $\left[\dot{\mathcal{H}}_{0}+\dot{\mathcal{H}}_{p}\right]-2\left[\mathcal{C}_{0}+\mathcal{C}_{p}\right]$ is skew-symmetric.

Proof:

(1) The first property can be shown easily by using the fact that

$$
\mathbf{W}^{T}=\mathrm{S} .
$$

(2) By the definitions of $\mathcal{H}_{0}, \mathcal{H}_{p}, \mathcal{C}_{0}$ and $\mathcal{C}_{p}$, it follows that

$$
\begin{aligned}
{\left[\dot{\mathcal{H}}_{0}+\dot{\mathcal{H}}_{p}\right] } & -2\left[\mathcal{C}_{p}+\mathcal{C}_{0}\right] \\
& =N_{1}+N_{2}+N_{3}+N_{4}+N_{5}
\end{aligned}
$$

where

$$
\begin{aligned}
& N_{1}=A^{T} \mathbf{W} \mathbf{J}^{-T}(\dot{\mathbf{H}}-2 \mathbf{C}) \mathbf{J}^{-1} \mathbf{S} A \\
& N_{2}=A^{T} \mathbf{W} \mathbf{J}^{-T} \mathbf{H} \mathbf{J}^{-1} \mathbf{S} A-A^{T} \mathbf{W} \mathbf{J}^{-T} \mathbf{H} \mathbf{J}^{-1} \mathbf{S} A, \\
& N_{3}=A^{T} \mathbf{W} \mathbf{J}^{-T} \mathbf{H} \mathbf{J}^{-1} \mathbf{S} A-A^{T} \mathbf{W} \mathbf{J}^{-T} \mathbf{H} \mathbf{J}^{-1} \dot{\mathbf{S}} A, \\
& N_{4}=\dot{A}^{T} \mathbf{W} \mathbf{J}^{-1} \mathbf{H J}^{-1} \mathbf{S} A-A^{T} \mathbf{W J}^{-T} \mathbf{H} \mathbf{J}^{-1} \mathbf{S} \dot{A}
\end{aligned}
$$

and

$$
N_{\mathbf{5}}=\left[\begin{array}{cc}
0 & 0 \\
0 & \mathcal{N}
\end{array}\right]
$$

where

$$
\begin{aligned}
\mathcal{N}= & \frac{d}{d t}\left(T_{0}^{T} R I_{0} R^{T} T_{0}\right) \\
& -2 T_{0}^{T} \omega_{0} \times R I_{0} R^{T} T_{0}-2 T_{0}^{T} R I_{0} R^{T} \dot{T}_{0}
\end{aligned}
$$

Property 2.1 ensures that the $N_{i}(i=1,2,3,4)$ are skew-symmetric. Furthermore it can be shown that $N_{5}$ is also skew-symmetric (see Appendix). Thus the result follows. 


\subsection{Controller Design}

Instead of designing control torque $\mathbf{T}$ directly, the transformed input $\mathcal{F}$ is specified first. This approach allows the use of the regularized model and its properties, as stated in Theorem 3.1. Section 6 introduces a method to calculate $\mathbf{T}$ from $\mathcal{F}$.

\section{Robust Control I}

To develop the first robust strategy, the decomposition of the transformed model is performed first, so that the payload parameters are isolated from the dynamics. To this end, the following matrices are introduced:

$$
\begin{aligned}
& E_{x x}=R\left[\begin{array}{lll}
1 & 0 & 0 \\
0 & 0 & 0 \\
0 & 0 & 0
\end{array}\right] R^{T}, \\
& E_{x y}=R\left[\begin{array}{lll}
0 & 1 & 0 \\
1 & 0 & 0 \\
0 & 0 & 0
\end{array}\right] R^{T}, \\
& E_{x z}=R\left[\begin{array}{lll}
0 & 0 & 1 \\
0 & 0 & 0 \\
1 & 0 & 0
\end{array}\right] R^{T}, \\
& E_{y y}=R\left[\begin{array}{lll}
0 & 0 & 0 \\
0 & 1 & 0 \\
0 & 0 & 0
\end{array}\right] R^{T}, \\
& E_{z z}=R\left[\begin{array}{lll}
0 & 0 & 0 \\
0 & 0 & 0 \\
0 & 0 & 1
\end{array}\right] R^{T}
\end{aligned}
$$

and

$$
E_{y z}=R\left[\begin{array}{lll}
0 & 0 & 0 \\
0 & 0 & 1 \\
0 & 1 & 0
\end{array}\right] R^{T}
$$

Therefore the matrix $R I_{0} R^{T}$ can be written as

$$
\begin{aligned}
R I_{0} R^{T} & =R\left[\begin{array}{lll}
I_{0 x x} & I_{0 x y} & I_{0 x z} \\
I_{0 y x} & I_{0 y y} & I_{0 y z} \\
I_{0 x x} & I_{0 z y} & I_{0 z z}
\end{array}\right] R^{T} \\
& =\sum_{i=x}^{z} \sum_{j=i}^{z} I_{0 i j} E_{i j}
\end{aligned}
$$

due to the symmetry of $I_{0}$. The transformed model (33) can now be decomposed as

$$
\begin{aligned}
\mathcal{F}= & \mathcal{H}_{0} \ddot{X}_{0}+\mathcal{C}_{0} \dot{X}_{0}+\mathcal{G}_{0} \\
& +\left[\begin{array}{c}
m\left(\ddot{x}_{0}+g\right) \\
\sum_{i=x}^{z}\left(\sum_{j=i}^{x} \mathcal{M}_{i j}\right.
\end{array}\right],
\end{aligned}
$$

where

$$
\begin{aligned}
\mathcal{M}_{i j}= & I_{0 i j} T_{0}^{T}\left[E_{i j} T_{0} \ddot{\phi}_{0}\right. \\
& \left.+\left(\omega_{0} \times E_{i j} T_{0}+E_{i j} \dot{T}_{0}\right) \dot{\phi}_{0}\right] .
\end{aligned}
$$

Based on this model, a robust path tracking control scheme is developed.

The control input $(\mathcal{F})$ is designed as

$$
\begin{aligned}
\mathcal{F}= & \mathcal{H}_{0}\left[\ddot{X}_{0}^{d}-(\lambda+\beta) \dot{\varepsilon}-\lambda \beta \varepsilon\right]+\mathcal{C}_{0}\left[\dot{X}_{0}^{d}-\beta \varepsilon\right] \\
& +\mathcal{G}_{0}+\left[\sum_{i=x}^{z} \sum_{j=i}^{z} \Psi_{0 i j} \Psi_{i j}\right]-K \Phi,
\end{aligned}
$$

where $\beta>0, \lambda>0$, and $K=K^{T}>0$ are design parameters (affecting stability, speed of response and disturbance rejection properties) and $\hat{m}$ and $I_{0}$ are computed by

$$
\begin{aligned}
\hat{I}_{0} & =\left[\begin{array}{lll}
\hat{I}_{0 x x} & \hat{I}_{0 x y} & \hat{I}_{0 x z} \\
\hat{I}_{0 x y} & \hat{I}_{0 y y} & \hat{I}_{0 y z} \\
\hat{I}_{0 x z} & \hat{I}_{0 y z} & \hat{I}_{0 z z}
\end{array}\right], \\
\hat{m} & =-\frac{\Phi_{1}^{T} \Psi_{m} \bar{m}^{2}}{\left|\Phi_{1}^{T} \Psi_{m}\right| \bar{m}^{2}+\nu} \\
\hat{I}_{0 x x} & =-\frac{\Phi_{2}^{T} \Psi_{x x} \bar{I}_{0 x x}^{2}}{\left|\Phi_{2}^{T} \Psi_{x x}\right| \bar{I}_{0 x x}+\nu}, \\
\hat{I}_{0 x y} & =-\frac{\Phi_{2}^{T} \Psi_{x y} \bar{I}_{0 x y}^{2}}{\left|\Phi_{2}^{T} \Psi_{x y}\right| \bar{I}_{0 x y}+\nu} \\
\hat{I}_{0 x z} & =-\frac{\Phi_{2}^{T} \Psi_{x z} \bar{I}_{0 x z}^{2}}{\left|\Phi_{2}^{T} \Psi_{x z}\right| \bar{I}_{0 x z}+\nu} \\
\hat{I}_{0 y y} & =-\frac{\Phi_{2}^{T} \Psi_{y y} \bar{I}_{0 y y}^{2}}{\left|\Phi_{2}^{T} \Psi_{y y}\right| \bar{I}_{0 y y}+\nu} \\
\hat{I}_{0 y z} & =-\frac{\Phi_{2}^{T} \Psi_{y z} \bar{I}_{0 y z}^{2}}{\left|\Phi_{2}^{T} \Psi_{y z}\right| \bar{I}_{0 y z}+\nu}
\end{aligned}
$$

and

$$
\hat{I}_{0 z z}=-\frac{\Phi_{2}^{T} \Psi_{z z} \bar{I}_{0 z z}^{2}}{\left|\Phi_{2}^{T} \Psi_{z z}\right| \bar{I}_{0 z z}+\nu}
$$

In (44)-(52), $\bar{m}$ and $\bar{I}_{0 i j}$ are the upper bounds of $m$ and $I_{0 i j}$, respectively,

$$
\begin{aligned}
\boldsymbol{\Psi}_{m} & =\ddot{x}_{0}^{d}-(\lambda+\beta) \dot{\varepsilon}_{1}-\lambda \beta \varepsilon_{1}+g \\
\boldsymbol{\Psi}_{i j} & =T_{0}^{T}\left\{E_{i j} T_{0}\left[\ddot{\phi}_{0}^{d}-(\lambda+\beta) \dot{\varepsilon}_{2}-\lambda \beta \varepsilon_{2}\right]\right. \\
& \left.+\left[\omega_{0} \times E_{i j} T_{0}+E_{i j} \dot{T}_{0}\right]\left(\dot{\phi}_{0}^{d}-\lambda \varepsilon_{2}\right)\right\} \\
\boldsymbol{\Phi}_{i} & =\dot{\varepsilon}_{i}+\beta \varepsilon_{i}, \quad i=1,2, \\
\boldsymbol{\Phi} & =\left[\boldsymbol{\Phi}_{1}^{T}, \boldsymbol{\Phi}_{2}^{T}\right]^{T}
\end{aligned}
$$

and $\nu \geq 0$ is a design variable that satisfies

$$
\int_{0}^{t} \nu(\tau) d \tau \leq C_{02}^{2}<\infty
$$

\section{Robust Control II}


For brevity, let

$$
\begin{aligned}
& \mathcal{Z}_{1}=\ddot{X}_{0}^{d}-(\lambda+\beta) \dot{\varepsilon}-\lambda \beta \varepsilon \\
& \mathcal{Z}_{2}=\dot{X}_{0}^{d}-\beta \varepsilon
\end{aligned}
$$

and

$$
L=\mathcal{H}_{p} \mathcal{Z}_{1}+\mathcal{C}_{p} \mathcal{Z}_{2}+\mathcal{G}_{p} .
$$

The control torque is specified by

$$
\mathcal{F}=\mathcal{H}_{0} \mathcal{Z}_{1}+\mathcal{C}_{0} \mathcal{Z}_{2}+\mathcal{G}_{0}-K \Phi+U_{a}
$$

and

$$
U_{a}=-\frac{\Phi \eta^{2}}{\|\Phi\| \eta+\nu}
$$

where $\eta$ is defined by

$$
\begin{aligned}
\left\|\mathcal{H}_{p}\right\|\left\|\mathcal{Z}_{1}\right\| & +\left\|\mathcal{C}_{p}\right\|\left\|\mathcal{Z}_{2}\right\|+\left\|\mathcal{G}_{p}\right\| \\
& \leq \alpha_{1}\|\mathcal{Z}\|+\alpha_{2}\left\|\dot{X}_{0}\right\|\left\|\mathcal{Z}_{2}\right\|+\alpha_{3} \\
& \triangleq \eta .
\end{aligned}
$$

In (61), the $\alpha_{i}$ represent upper bounds on the model norms.

The proposed control strategies lead to the following results.

\section{Theorem 3.2}

Consider the multi-robot dynamics (33) in which the payload parameters $m$ and $I_{0}$ are unknown a priori. If the control input $\mathcal{F}$ is designed as in (44) $-(57)$, the payload then asymptotically tracks the given position and orientation, i.e., $X_{0} \rightarrow X_{0}^{d}$ and $\dot{X}_{0} \rightarrow \dot{X}_{0}^{d}$ as $t \rightarrow \infty$.

\section{Theorem 3.3}

Given the conditions stated in Theorem 3.2, if the control input $\mathcal{F}$ is specified as in (58) and (59), then stable path tracking of payload is ensured.

\section{STABILITY}

Stability can be proven by the following result (see [25] for more detail). Consider a dynamical system $\Sigma(\xi, \dot{\xi})$ with $\xi \in R^{n}$ being the system state. Defining the generalized energy function of the system as

$$
E(\xi)=\xi^{T} K \xi,
$$

where $K \in R^{n \times n}$ is a symmetric positive definite matrix, leads to the following result.

\section{Theorem 4.1}

Let $J_{A}$ be the integration of the generalized energy function $E(\xi)$ over the time interval $[0, t]$, i.e., $J_{A}=\int_{0}^{t} E(\xi) d \tau$. Suppose $\xi$ is uniformly continuous. If $J_{A} \leq C_{0}^{2}<\infty$ for all $t \in[0, \infty)$, then the system is asymptotically stable, i.e., $\xi \rightarrow 0$ as $t \rightarrow \infty$.

This result can be shown by using Barbalat's Lemma [20] (see [25] for details).

The interpretation of the above theorem is that the system must be stable if the accumulation of the system's energy over a time interval of infinite length is finite. One advantage to this approach is that it is fairly easy to choose a suitable energy function. (Another advantage is discussed later.) In the following, this result is used to prove the tracking stability of the proposed strategies.

\section{Proof of Theorem 3.2:}

Note that if the control input in (33) is designed as in (44), the closed-loop system dynamics is given by

$$
\begin{aligned}
K \Phi= & -\left(\mathcal{H}_{0}+\mathcal{H}_{p}\right)(\dot{\Phi}+\lambda \Phi)-\left(\mathcal{C}_{0}+\mathcal{C}_{p}\right) \Phi \\
& +\left[\begin{array}{c}
(\hat{m}-m) \Psi_{m} \\
\sum_{i=x}^{z} \sum_{j=i}^{z}\left(\hat{I}_{0 i j}-I_{0 i j}\right) \Psi_{i j}
\end{array}\right] .
\end{aligned}
$$

To simplify notation in the following, let $H^{*}=\mathcal{H}_{0}+$ $\mathcal{H}_{p}$ and $C^{*}=\mathcal{C}_{0}+\mathcal{C}_{p}$. As can be seen, there exist several generalized energy functions for this problem. For example

$$
\begin{aligned}
E_{1}(\Phi) & =\Phi^{T} K \Phi \\
E_{2}(\lambda, \Phi) & =\lambda \Phi^{T} H^{*} \Phi \quad(\forall \lambda>0)
\end{aligned}
$$

or

$$
E_{3}(\lambda, \Phi)=\Phi^{T} K \Phi+\lambda \Phi^{T} H^{*} \Phi \quad(\forall \lambda>0) .
$$

In the following only $E_{1}(\Phi)$ is considered. Clearly

$$
\begin{aligned}
J_{A}= & \int_{0}^{t} E_{1}(\Phi) d \tau \\
= & \int_{0}^{t} \Phi^{T} K \Phi d \tau \\
= & -\int_{0}^{t} \Phi^{T} H^{*}(\dot{\Phi}+\lambda \Phi) d \tau-\int_{0}^{t} \Phi^{T} C^{*} \Phi d \tau \\
& +\int_{0}^{t}(\hat{m}-m) \Phi_{1}^{T} \Psi_{m} d \tau \\
& +\sum_{i=x}^{z} \sum_{j=i}^{z} \int_{0}^{t}\left(\hat{I}_{0 i j}-I_{0 i j}\right) \Phi_{2}^{T} \Psi_{i j} d \tau .
\end{aligned}
$$

Noting that $H^{*}$ is symmetric positive definite, it can be shown that

$$
\lambda \int_{0}^{i} \Phi^{T} H^{*} \Phi d \tau>0 \quad(\forall \lambda>0)
$$


and

$$
\int_{0}^{t} \Phi^{T} H^{*} \dot{\Phi} d \tau \geq-\left.\frac{1}{2} \Phi^{T} H_{-}^{*} \Phi\right|_{t=0}-\frac{1}{2} \int_{0}^{t} \Phi^{T} \dot{H}^{*} \Phi d \tau
$$

Using these relations yields the inequality

$$
\begin{aligned}
J_{A} \leq & C_{01}^{2}+\int_{0}^{t}(\hat{m}-m) \Phi_{1}^{T} \Psi_{m} d \tau \\
& +\sum_{i=x}^{z} \sum_{j=i}^{z} \int_{0}^{t}\left(\hat{I}_{0 i j}-I_{0 i j}\right) \Phi_{2}^{T} \Psi_{i j} d \tau \\
& +\frac{1}{2} \int_{0}^{t} \Phi^{T}\left(\dot{H}^{*}-2 C^{*}\right) \Phi d \tau
\end{aligned}
$$

where $C_{01}^{2}=\left.\frac{1}{2} \Phi^{T} H^{*} \Phi\right|_{t=0}$. Due to the property of skew-symmetry, $\int_{0}^{t} \Phi^{T}\left(\dot{H}^{*}-2 C^{*}\right) \Phi d \tau=0$. Employing this and the bounds on $m$ and $I_{0},(62)$ becomes

$$
\begin{aligned}
J_{A} \leq & C_{01}^{2}+\int_{0}^{t}(\hat{m}-m) \Phi_{1}^{T} \Psi_{m} d \tau \\
& +\sum_{i=x}^{z} \sum_{j=i}^{z} \int_{0}^{t}\left(\hat{I}_{0 i j}-I_{0 i j}\right) \Phi_{2}^{T} \Psi_{i j} d \tau \\
\leq & C_{01}^{2}+\int_{0}^{t} \hat{m} \Phi_{1}^{T} \Psi_{m} d \tau+\int_{0}^{t} \bar{m}\left|\Phi_{1}^{T} \Psi_{m}\right| d \tau \\
& +\sum_{i=x}^{z} \sum_{j=i}^{z} \int_{0}^{t} \hat{I}_{0 i j} \Phi_{2}^{T} \Psi_{i j} d \tau \\
& +\sum_{i=x}^{z} \sum_{j=i}^{z} \int_{0}^{t} \bar{I}_{0 i j}\left|\Phi_{2}^{T} \Psi_{i j}\right| d \tau .
\end{aligned}
$$

Inserting the algorithms (46)-(52) into (63) and conducting a little manipulation yields

$$
\begin{aligned}
J_{A} \leq & C_{01}^{2}+\int_{0}^{t} \frac{\left|\Phi_{1}^{T} \Psi_{m}\right| \bar{m}_{\nu}}{\left|\Phi_{1}^{T} \Psi_{m}\right| \bar{m}+\nu} d \tau \\
& +\sum_{i=x}^{z} \sum_{j=i}^{z} \int_{0}^{t} \frac{\left|\Phi_{2}^{T} \Psi_{i j}\right| \bar{I}_{0 i j} \nu}{\left|\Phi_{2}^{T} \Psi_{i j}\right| \bar{I}_{0 i j}+\nu} d \tau \\
\leq & C_{01}^{2}+\int_{0}^{t} \nu d \tau+\sum_{i=x}^{z} \sum_{j=i}^{z} \int_{0}^{t} \nu d \tau
\end{aligned}
$$

Since $\nu$ satisfies (57),

$$
J_{A} \leq C_{01}^{2}+7 C_{02}^{2}<\infty,
$$

which implies that $\Phi \in L_{2}$. Furthermore, by making use of the same argument as in Song and Middleton [26], it can be shown that $\Phi \in L_{\infty}$ and $\dot{\Phi} \in L_{\infty}$. Hence $\Phi$ is uniformly continuous. By Theorem $4.1, \Phi \rightarrow 0$ as $t \rightarrow \infty$. Since $\Phi=\dot{\varepsilon}+\lambda \varepsilon$, the result stated in Theorem 3.2 is obtained.

\section{Proof of Theorem 3.3}

In this case, the control law (58)-(60) yields the closed-loop dynamics

$$
\left(\mathcal{H}_{0}+\mathcal{H}_{p}\right)(\dot{\Phi}+\lambda \Phi)+\left(\mathcal{C}_{0}+\mathcal{C}_{p}\right) \Phi=-K \Phi+U_{a}-L,
$$

where $L$ is defined as before. Following the same procedure as in the proof of Theorem 3.2, it is seen that

$$
\begin{aligned}
J_{A} & =\int_{0}^{t} \Phi^{T} K \Phi d \tau \\
& \leq C_{01}^{2}+\int_{0}^{t} \Phi^{T}\left[U_{a}-L\right] d \tau \\
& \leq C_{01}^{2}+\int_{0}^{t} \Phi^{T} U_{a} d \tau+\int_{0}^{t}\|\Phi\|\|L\| d \tau .
\end{aligned}
$$

Substituting for $U_{a}$ from (59) and noting that $\|L\| \leq \eta$ (see (60)), yields

$$
\begin{aligned}
J_{A} & \leq C_{01}^{2}-\int_{0}^{t} \frac{\|\Phi\|^{2} \eta^{2}}{\|\Phi\| \eta+\nu} d \tau+\int_{0}^{t}\|\Phi\| \eta d \tau \\
& =C_{01}^{2}+\int_{0}^{t} \frac{\|\Phi\| \eta \nu}{\|\Phi\| \eta+\nu} d \tau \\
& \leq C_{01}^{2}+\int_{0}^{t} \nu d \tau .
\end{aligned}
$$

Since $\nu$ satisfies (57), $J_{A}$ is bounded and the result follows from Theorem 4.1.

\section{Remark 4.1}

It is seen that the design variable $\nu$ plays an important role in the above control strategies. It is required in both that $\nu$ be integrable. As can be verified,

$$
\nu=\frac{1}{2} v_{1}\left(1+t^{m}\right)^{\rho} e^{-v_{2} t^{n}}
$$

where $v_{1} \geq 0$ and $v_{2} \geq 0$, satisfies such a requirement if $m, \rho$ and $n$ are chosen properly.

\section{Remark 4.2}

It is worth mentioning that, in addition to proving stability, $J_{\boldsymbol{A}}$ also provides a relative measure of the tracking performance in terms of transient and steady-state errors. This is because $\Phi$ is a filtered tracking error, and the quantity $\Phi^{T} h \Phi$ is a weighted version of the squared error. Its integral over $\left[t_{0}, t\right]$ represents the accumulation of the weighted, squared tracking error within the interval. The smaller this integral, the better the tracking performance. (See [25] for a thorough investigation of this point.) 


\section{SIMPLIFIED STRATEGÝ}

In strategy II, the quantity $\eta$ has to be determined. This is accomplished by estimating $\alpha_{1}, \alpha_{2}$ and $\alpha_{3}$. Knowing the bounds on $m$ and $I_{0}$ and the formulations of $\mathcal{H}_{p}, \mathcal{C}_{p}$ and $\mathcal{G}_{p}$ makes it possible to obtain these quantities. To eliminate this tedious procedure, an alternate control strategy is proposed.

\section{Theorem 5.1}

Let the control input be defined as

$$
\mathcal{F}=\mathcal{H}_{0} \mathcal{Z}_{1}+\mathcal{C}_{0} \mathcal{Z}_{2}+\mathcal{G}_{0}-K \Phi+U_{a}
$$

with $\mathcal{Z}_{1}$ and $\mathcal{Z}_{2}$ defined as before and

$$
U_{a}=-\frac{\Phi \hat{\eta}}{\|\Phi\|}
$$

where

$$
\hat{\eta}=\hat{\alpha}_{1}\left\|\mathcal{Z}_{1}\right\|+\hat{\alpha}_{2}\left\|\dot{X}_{0}\right\|\left\|\mathcal{Z}_{2}\right\|+\hat{\alpha}_{3} .
$$

If the $\hat{\alpha}_{i}$ are estimated on-line via

$$
\begin{aligned}
& \hat{\alpha}_{1}=\hat{\alpha}_{1}(0)+c_{1} \int_{0}^{t}\|\Phi\|\left\|\mathcal{Z}_{1}\right\| d \tau, \\
& \hat{\alpha}_{2}=\hat{\alpha}_{2}(0)+c_{2} \int_{0}^{t}\|\Phi\|\left\|\dot{X}_{0}\right\|\left\|\mathcal{Z}_{2}\right\| d \tau,
\end{aligned}
$$

and

$$
\hat{\alpha}_{3}=\hat{\alpha}_{3}(0)+c_{3} \int_{0}^{t}\|\Phi\| d \tau,
$$

where $\hat{\alpha}_{i}(0)$ is the initial value of $\hat{\alpha}_{i}$ and the $c_{i}$ are positive constants, then stable path tracking is achieved.

Proof:

Applying this strategy to (33) leads to the following.

$$
\begin{aligned}
J_{A}= & \int_{0}^{t} \Phi^{T} K \Phi d \tau \\
\leq & C_{01}^{2}+\int_{0}^{t}\|\Phi\|(\eta-\hat{\eta}) d \tau \\
= & C_{01}^{2}+\int_{0}^{t}\left(\alpha_{1}-\hat{\alpha}_{1}\right)\|\Phi\|\left\|\mathcal{Z}_{1}\right\| d \tau \\
& +\int_{0}^{t}\left(\alpha_{2}-\hat{\alpha}_{2}\right)\|\Phi\|\left\|\dot{X}_{0}\right\|\left\|\mathcal{Z}_{2}\right\| d \tau \\
& +\int_{0}^{t}\left(\alpha_{3}-\hat{\alpha}_{3}\right)\|\Phi\| d \tau .
\end{aligned}
$$

To show the boundedness of $J_{A}$, the following relation is needed,

$$
\int_{0}^{t} \int_{0}^{\tau} f(\gamma) d \gamma f(\tau) d \tau=\frac{1}{2}\left(\int_{0}^{t} f(\tau) d \tau\right)^{2}
$$

Substituting for the $\hat{\alpha}_{i}$ in (67) and using the above relation yields

$$
\begin{aligned}
J_{A} \leq & C_{01}^{2}+\left(\alpha_{1}-\hat{\alpha}_{1}(0)\right) \int_{0}^{t}\|\Phi\|\left\|\mathcal{Z}_{1}\right\| d \tau \\
& -\frac{c_{1}}{2}\left[\int_{0}^{t}\|\Phi\|\left\|\mathcal{Z}_{1}\right\| d \tau\right]^{2} \\
& +\left(\alpha_{2}-\hat{\alpha}_{2}(0)\right) \int_{0}^{t}\|\Phi\|\left\|\dot{X}_{0}\right\|\left\|\mathcal{Z}_{2}\right\| d \tau \\
& -\frac{c_{2}}{2}\left[\int_{0}^{t}\|\Phi\|\left\|\dot{X}_{0}\right\|\left\|\mathcal{Z}_{2}\right\| d \tau\right]^{2} \\
& +\left(\alpha_{3}-\hat{\alpha}_{3}(0)\right) \int_{0}^{t}\|\Phi\| d \tau \\
& -\frac{c_{3}}{2}\left[\int_{0}^{t}\|\Phi\| d \tau\right]^{2} .
\end{aligned}
$$

By completing the square,

$$
\begin{aligned}
J_{A} \leq & C_{01}^{2}+\frac{\left(\alpha_{1}-\hat{\alpha}_{1}(0)\right)^{2}}{2 c_{1}}+\frac{\left(\alpha_{2}-\hat{\alpha}_{2}(0)\right)^{2}}{2 c_{2}} \\
& +\frac{\left(\alpha_{3}-\hat{\alpha}_{3}(0)\right)^{2}}{2 c_{3}} \triangleq C_{0}^{2}<\infty .
\end{aligned}
$$

The result follows using the same argument as before.

\section{Remark 5.1}

The primary advantage of this strategy is that one does not need to calculate the design parameters $\alpha_{1}, \alpha_{2}$, and $\alpha_{3}$. Instead, these variables are updated on-line using simple algorithms.

\section{TASK DISTRIBUTION}

What is actually needed to guarantee the path tracking of the multi-robot/payload system is the vector of control torques for each robot (the elements of $T$ ). Fortunately, since $\mathbf{W}$ is full rank (Property 2.1 ), there exists a matrix,

$$
\mathbf{W}^{+}=\mathbf{W}^{T}\left(\mathbf{W} \mathbf{W}^{T}\right)^{-1} \in R^{6 d \times 6},
$$

such that the total control torque $\mathbf{T}$ becomes

$$
\mathbf{T}=\mathbf{J}^{T} \mathbf{F}_{\text {end }},
$$

where

$$
\begin{aligned}
\mathbf{F}_{\text {end }} & =\mathbf{W}^{+} A^{-T} \mathcal{F}+\mathbf{F}_{I} \\
& \triangleq \mathbf{F}_{P}+\mathbf{F}_{I}
\end{aligned}
$$

In these equations, $\mathbf{F}_{P}$ is the force causing the motion of the payload ( $\mathcal{F}$ is computed by (44), (58) and (59), 
or (64)-(66)) and $\mathbf{F}_{I} \in$ Null Space(W) represents an internal force vector. A well known formulation for $\mathbf{F}_{I}$,

$$
\mathbf{F}_{I}=\left(E_{6 d}-\mathbf{W}^{+} \mathbf{W}\right) \mu^{-} \quad \forall \mu \in R^{6 d},
$$

has been the basis for much work dealing with load distribution. However, it is noted in [34] that, although $\mathbf{F}_{I}$ in (69) satisfies $\mathbf{W} \mathbf{F}_{I}=0$, it is an inadequate definition for internal force from a physical point of view. A recent work [31] also pointed out that (69) does not completely define the internal loading.

Furthermore, in practical applications force constraints are generally imposed on the manipulating forces/moments at the grasp points due to the limited control energy, i.e.,

$$
\begin{aligned}
\left|F_{\text {end }}(i)\right|= & \left|F_{P_{j}}(i)+F_{I_{j}}(i)\right| \leq \mu_{j}(i), \\
& j=1, \ldots, d, \quad i=1, \ldots, 6,
\end{aligned}
$$

where $F_{\text {end }}(i), F_{P_{j}}(i)$ and $F_{I_{j}}(i)$ are the $i^{\text {th }}$ elements of the $j^{\text {th }}$ partitions of $\mathbf{F}_{\text {end }}, \mathbf{F}_{P}$ and $\mathbf{F}_{I}$, respectively, and $\mu_{j}(i)$ are given positive numbers. Such constraints are also necessary to achieve fine manipulation.

So an interesting problem is how to distribute the task among the robots such that the force constraints (70) and $\mathbf{W F}_{I}=0$ are satisfied. The following strategy provides a solution to this problem.

First let

$$
\begin{aligned}
& \Omega_{1} \triangleq \quad \text { the set of robots working on the task } \\
& \Omega_{2} \triangleq \quad \text { the set of robots needing help\}, and } \\
& \Omega_{3} \triangleq \text { \{the set of robots with spare capacity. }
\end{aligned}
$$

Assume $\Omega_{1}=\Omega_{2} \oplus \Omega_{3}$ and $\Omega_{2} \neq\{\theta\}$ and $\Omega_{3} \neq\{\theta\}$. This implies that each robot either needs help or has spare capacity, at least one robot needs help and at least one robot can provide help. It is further assumed that the number of the robots with spare capacity is $r$ and those robots are able to provide the required forces.

The strategy basically consists of two steps. Step 1 checks which robots need help and step 2 arranges the help. The first step uses $F_{P_{j}}(i)$ as a criterion. That is,

- STEP 1a: If $\left|F_{P_{j}}(i)\right| \geq \mu_{j}(i)$, then $j \in \Omega_{2}$.

- STEP 1b: The $F_{I_{j}}$ are adjusted so as to guarantee the force constraints (70). This is achieved by choosing $F_{I_{j}}$ as

$$
\begin{aligned}
F_{I_{j}}(i) & \triangleq F_{I_{j}}^{*}(i) \\
& = \begin{cases}\mu_{j}(i)-F_{P_{j}}(i), & \text { if } F_{P_{j}}(i) \geq \mu_{j}(i) ; \\
-\mu_{j}(i)-F_{P_{j}}(i), & \text { if } F_{P_{j}}(i) \leq-\mu_{j}(i) .\end{cases}
\end{aligned}
$$

The second step is motivated by the following observations. First it is noted that in order to make the payload asymptotically track the desired path, the total control force $\mathcal{F}$ must be equivalently generated by the total joint torque. Thus $\mathbf{T}$ should satisfy,

$$
A^{T} \mathbf{W J}^{-T} \mathbf{T}=A^{T} \mathbf{W F}_{\text {end }}=\mathcal{F} \text {. }
$$

With $F_{I}$, specified as in (71), the condition (72) may not be satisfied. Furthermore, (71) may also cause the null space property of $\mathbf{F}_{I}$ to be violated. Hence we need to seek help from the other robots. Clearly such help should completely compensate the load that the robot $j\left(j \in \Omega_{2}\right)$ cannot supply. This is ensured if the payload lies within the loading capacity of the robots (otherwise, more robots should be assigned to the task). Once $F_{I_{j}}\left(j \in \Omega_{2}\right)$ is specified according to $(71), F_{I_{k}}\left(k \in \Omega_{3}\right)$ must be chosen such that the null space condition holds. This is ensured if $F_{I_{k}}$ is determined by

$$
\sum_{k=k_{1}}^{k_{r}} W_{k} F_{I_{k}}=-\sum_{\text {all }} W_{j \in \Omega_{2}} F_{I_{j}}^{*},
$$

where $F_{I_{j}}^{*}$ is given by (71).

In helping robot $j\left(j \in \Omega_{2}\right)$, there is no constraint on how much effort each robot in $\Omega_{3}$ should provide. Hence one generally has infinite choices for $F_{I_{k}}$, as long as the resultant $\mathbf{F}_{I}$ lies in the null space of $\mathbf{W}$. But what we are interested in is an "optimal" choice for the $F_{I_{k}}$. This brings us to step 2 .

- STEP 2: Determine the $F_{I_{k}}(i), i=1, \ldots, 6, k \in \Omega_{3}$, such that, under the constraints (70) and (73), the cost function,

$$
J_{c}\left(F_{I_{k}}\right)=\frac{1}{2} \sum_{k=k_{1}}^{k_{r}} \sum_{i=1}^{6} \rho_{k}(i) F_{I_{k}}^{2}(i)=\frac{1}{2} \chi^{T} P \chi,
$$

is minimized. In this equation, $\rho_{k}(i)>0$ is a weighting parameter, $\chi=\left[\begin{array}{ll}F_{I_{k_{2}}}^{T} & F_{I_{k_{2}}}^{T} \ldots F_{I_{k_{r}}}^{T}\end{array}\right]^{T} \in R^{6 r}$ and $P=$ $\operatorname{diag}\left[\rho_{k}(i)\right] \in R^{6 r \times 6 r}$ is a symmetric, positive-definite matrix.

By denoting

$$
\Gamma=-\sum_{\text {all } j \in \Omega_{3}} W_{j} F_{I_{j}}^{*} \in R^{6}
$$

the constraints (73) can be rewritten as

$$
Q \chi=\Gamma \text {, }
$$

where $Q=\left[\begin{array}{lll}W_{k_{1}} & W_{k_{2}} \ldots W_{k_{r}}\end{array}\right] \in R^{6 \times 6 r}$. Therefore, the optimal task distribution problem under force constraints becomes

$$
\begin{aligned}
\text { minimize: } & J_{c}(\chi)=\frac{1}{2} \chi^{T} P \chi \\
\text { subject to: } & Q \chi=\Gamma .
\end{aligned}
$$


The Lagrangian multiplier method is used to solve this problem. Using $\nu_{i}, i=1, \ldots, 6$ as the Lagrange multipliers, the Lagrangian function is

$$
\begin{aligned}
L(\chi, \nu) & =J_{c}(\chi)+\sum_{i=1}^{6} \nu_{i}\left[(Q \chi)_{i}-\Gamma_{i}\right] \\
& =\frac{1}{2} \chi^{T} P \chi+\nu^{T}[Q \chi-\Gamma]
\end{aligned}
$$

The necessary conditions for the optimal solution can be found from

$$
\begin{aligned}
\frac{\partial L(\chi, \nu)}{\partial \chi} & =\frac{\partial\left[\frac{1}{2} \chi^{T} P \chi\right]}{\partial \chi}+\frac{\partial\left[\nu^{T} Q \chi\right]}{\partial \chi} \\
& =P \chi+Q^{T} \nu=0
\end{aligned}
$$

and

$$
\frac{\partial L(\chi, \nu)}{\partial \nu}=Q \chi-\Gamma=0
$$

Thus

$$
\nu^{*}=-\left[Q P^{-1} Q^{T}\right]^{-1} \Gamma
$$

and

$$
\chi^{*}=P^{-1} Q^{T}\left[Q P^{-1} Q^{T}\right]^{-1} \Gamma .
$$

Correspondingly the minimium cost function is

$$
J_{c_{\text {optimel }}}=L\left(\chi^{*}, \nu^{*}\right)=\frac{1}{2} \Gamma^{T}\left[Q P^{-1} Q^{T}\right]^{-1} \Gamma .
$$

Notice that the inverse of the matrix $\left[Q P^{-1} Q^{T}\right]$ should exist in order to obtain (76), (77) and (78). Since this is an important issue concerning the existence of the optimal solution, a rigorous proof of the invertibility of the matrix $Q P^{-1} Q^{T}$ is worth investigating. For simplicity, let $P=E_{6 r}$, a unit matrix. In view of the definition of $Q$, it is seen that

$$
Q Q^{T}=W_{k_{1}} W_{k_{1}}^{T}+W_{k_{2}} W_{k_{2}}^{T}+\cdots+W_{k_{r}} W_{k_{r}}^{T} .
$$

Since

$$
W_{i}=\left[\begin{array}{cc}
E_{3} & 0 \\
B_{i} & E_{3}
\end{array}\right]
$$

where

$$
B_{i}=\left(R c_{i}\right) \times
$$

then

$$
W_{k_{i}} W_{k_{i}}^{T}=\left[\begin{array}{cc}
E_{3} & B_{k_{i}}^{T} \\
B_{k_{i}} & E_{3}+B_{k_{i}} B_{k_{i}}^{T}
\end{array}\right] .
$$

Therefore

$$
Q Q^{T}=\left[\begin{array}{cc}
r E_{3} & \sum_{i=k_{1}}^{k_{r}} B_{i}^{T} \\
\sum_{i=k_{1}}^{k_{r}} B_{i} & r E_{3}+\sum_{i=k_{1}}^{k_{r}} B_{i} B_{i}^{T}
\end{array}\right] .
$$

The Schur formula,

$$
\operatorname{det}\left[\begin{array}{ll}
A & B \\
C & D
\end{array}\right]=\operatorname{det}(A) \operatorname{det}\left(D-C A^{-1} B\right)
$$

gives

$$
\operatorname{det}\left(Q Q^{T}\right)=r \operatorname{det}\left[r E_{3}+\Lambda\right]
$$

where

$$
\Lambda=\sum_{i=k_{1}}^{k_{r}} B_{i} B_{i}^{T}-\frac{1}{r}\left[\sum_{i=k_{1}}^{k_{r}} B_{i}\right]\left[\sum_{i=k_{1}}^{k_{r}} B_{i}^{T}\right] .
$$

With a little manipulation, it can be shown that

$$
\begin{aligned}
{\left[\sum_{i=k_{1}}^{k_{r}} B_{i}\right]\left[\sum_{i=k_{1}}^{k_{r}} B_{i}^{T}\right] } & =\sum_{i=k_{1}}^{k_{r}} B_{i} B_{i}^{T} \\
& +\sum_{i=k_{1}}^{k_{r-1}} \sum_{j=i+1}^{k_{r}}\left(B_{i} B_{j}^{T}+B_{j} B_{i}^{T}\right) .
\end{aligned}
$$

This relation reduces $\Lambda$ to

$$
\begin{aligned}
\Lambda= & \frac{(r-1)}{r} \sum_{i=k_{1}}^{k_{r}} B_{i} B_{i}^{T} \\
& -\frac{1}{r} \sum_{i=k_{1}}^{k_{r-1}} \sum_{j=i+1}^{k_{r}}\left(B_{i} B_{j}^{T}+B_{j} B_{i}^{T}\right) .
\end{aligned}
$$

Also noting that

$$
(r-1) \sum_{i=k_{1}}^{k_{r}} B_{i} B_{i}^{T}=\sum_{i=k_{1}}^{k_{r-1}} \sum_{j=i+1}^{k_{r}}\left(B_{i} B_{i}^{T}+B_{j} B_{j}^{T}\right)
$$

$\forall r \geq 1$, it can be shown that

$$
\Lambda=\frac{1}{r} \sum_{i=k_{1}}^{k_{r-1}} \sum_{j=i+1}^{k_{r}}\left(B_{i}-B_{j}\right)\left(B_{i}-B_{j}\right)^{T},
$$

which shows that $\Lambda$ is at least positive semi-definite. Therefore $r E_{3}+\Lambda$ is positive definite and $Q Q^{T}$ is invertible. The same conclusion can be drawn for a general diagonal $P$ with more effort. Based on this discussion, following results can be claimed.

\section{Theorem 6.1}

If $\mathbf{T}$ is generated by

$$
\mathbf{T}=\mathbf{J}^{T} \mathbf{W}^{+} A^{-T} \mathcal{F}+\mathbf{J}^{T} \mathbf{F}_{I},
$$

where $\mathcal{F}$ is from (44), (58) and (59), or (64)-(66), $F_{I_{j}}\left(j \in \Omega_{2}\right)$ is specified by $(71)$ and $F_{I_{k}}\left(k \in \Omega_{3}\right)$ is computed by (77), then; 
(1) asymptotically stable path tracking is ensured,

(2) internal forces are non-zero at the contact points,

(3) force constraints are guaranteed and

(4) optimal sharing of the task is achieved.

Result 1 is true because such a $\mathbf{T}$ leads to the equivalent control force $\mathcal{F}$. Results 2,3 and 4 hold because the choice for $F_{I}$ satisfies (71), (73) and (77). The property of non-zero internal force is of particular interest in many advanced applications where no slippage and effective manipulation are required. It is seen that with this strategy, whenever $\left|F_{P_{j}}\right|>\mu_{j}(i)$, help from other robots is provided. Thus the given task is shared in a colleague-like manner in the sense that robots help each other when necessary. Furthermore, with $F_{I_{k}}\left(k \in \Omega_{3}\right)$ determined by (77), the task is shared among the robots in $\Omega_{3}$ optimally in that the cost function (74) is minimized.

\section{DESIGN EXAMPLE}

The case of three robots (each with three joints) transfering a point-mass payload is used to demonstrated the application of the strategy. Note that no rotations are involved in this case. Assume that the force constraints at the grasp point for robot $i(i=1,2,3)$ in the $x, y$ and $z$ directions are given in Newtons as

$$
\begin{aligned}
& \left|F_{\text {endi }}(x)\right| \leq 120 \\
& \left|F_{\text {endi }}(y)\right| \leq 150
\end{aligned}
$$

and

$$
\left|F_{\text {end, }}(z)\right| \leq 150 \text {. }
$$

Since the payload is a point-mass with no rotation, $A=$ $E_{3}$ and $\mathbf{W}=\left[\begin{array}{lll}E_{3} & E_{3} & E_{3}\end{array}\right]$. Hence

$$
W^{+}=\frac{1}{3}\left[\begin{array}{l}
E_{3} \\
E_{3} \\
E_{3}
\end{array}\right], \mathbf{F}_{P}=\frac{1}{3}\left[\begin{array}{l}
\mathcal{F} \\
\mathcal{F} \\
\mathcal{F}
\end{array}\right],
$$

where $\mathcal{F} \in R^{3}$ is computed by (44), (58) and (59), or (64)-(66).

Suppose that at time $t_{1}$,

$$
\begin{aligned}
& \left|F_{P_{1}}(x)\left(t_{1}\right)\right| \geq 120 \\
& \left|F_{P_{1}}(y)\left(t_{1}\right)\right| \geq 150
\end{aligned}
$$

and

$$
\left|F_{P_{1}}(z)\left(t_{1}\right)\right| \geq 150 .
$$

Then robot 1 needs help and the $F_{I_{1}}(x / y / z)$ are specified as

$$
F_{I_{1}}^{*}(x)= \begin{cases}120-\frac{1}{3} \mathcal{F}(x) & \text { if } \frac{1}{3} \mathcal{F}(x) \geq 120 \\ -120-\frac{1}{3} \mathcal{F}(x) & \text { if } \frac{1}{3} \mathcal{F}(x) \leq-120\end{cases}
$$

$$
F_{I_{1}}^{*}(y)= \begin{cases}150-\frac{1}{3} \mathcal{F}(y) & \text { if } \frac{1}{3} \mathcal{F}(y) \geq 150 \\ -150-\frac{1}{3} \mathcal{F}(y) & \text { if } \frac{1}{3} \mathcal{F}(y) \leq-150\end{cases}
$$

and

$$
F_{I_{1}}^{*}(z)= \begin{cases}150-\frac{1}{3} \mathcal{F}(z) & \text { if } \frac{1}{3} \mathcal{F}(z) \geq 150 \\ -150-\frac{1}{3} \mathcal{F}(z) & \text { if } \frac{1}{3} \mathcal{F}(z) \leq-150\end{cases}
$$

To optimally help robot 1 , choose $P=\frac{1}{4} E_{6}$. By (77) the task can be optimally shared if

$$
F_{I_{3}}^{*}=-\frac{1}{2} F_{I_{1}}^{*} \text { and } F_{I_{3}}^{*}=-\frac{1}{2} F_{I_{1}}^{*},
$$

and the minmium cost function is

$$
J_{c_{\text {optimal }}}=\frac{1}{16} F_{I_{1}}^{*}{ }^{T} F_{I_{1}}^{*} .
$$

It can be verified that;

(1) the force constraints are satisfied,

(2) the null space property holds,

$$
\begin{aligned}
\mathbf{W F}_{I} & =F_{I_{1}}+F_{I_{2}}+F_{I_{3}} \\
& =F_{I_{1}}^{*}-\frac{1}{2} F_{I_{1}}^{*}-\frac{1}{2} F_{I_{1}}^{*} \\
& =0
\end{aligned}
$$

and

(3) the equivalent control force $\mathcal{F}$ is guaranteed since

$$
\begin{aligned}
A^{T} \mathbf{W}\left(\mathbf{F}_{P}+\mathbf{F}_{I}\right)= & E_{3}^{T}\left[E_{3} E_{3} E_{3}\right]\left[\begin{array}{l}
F_{P_{1}}+F_{I_{1}} \\
F_{P_{2}}+F_{I_{2}} \\
F_{P_{3}}+F_{I_{3}}
\end{array}\right] \\
= & \left(F_{P_{1}}+F_{P_{2}}+F_{P_{3}}\right) \\
& +\left(F_{l_{1}}+F_{I_{2}}+F_{l_{3}}\right) \\
= & \left(\frac{1}{3} \mathcal{F}+\frac{1}{3} \mathcal{F}+\frac{1}{3} \mathcal{F}\right)+0 \\
= & \mathcal{F} .
\end{aligned}
$$

\section{CONCLUSIONS}

The path tracking control problem of a multi-robot system handling an unknown rigid payload is studied. Based on the combined dynamic model which reflects payload effects, three robust path tracking control algorithms are constructed. The payload can be of any shape as long as its center-of-mass is known. As can be seen, the strategies do not require wrist force sensors, but the quantity $\left\{\left(x_{0}, \phi_{0}\right),\left(\dot{x}_{0}, \dot{\phi}_{0}\right)\right\}$ is required. Also the matrix $S_{i}$, which depends on the location and orientation of the $i^{\text {th }}$ end-effector, is needed. A vision system would be appropriate to obtain this information.

Notice that in this work the manipulation force and moment constraints are explicitly considered. In some applications, it is desirable to limit the stress in the 
object while manipulating it. This imposes a constraint on the internal forces of the form,

$$
F_{I_{j}}(i) \dot{\epsilon} \Delta_{j}(\bar{i})
$$

where $\Delta_{j}(i)$ describes the region in which the $i^{\text {th }}$ element of the internal force at contact point $j$ should lie. This region is specified as

$$
\Delta_{j}(i)=\left[\eta_{j}^{-}(i), \eta_{j}^{+}(i)\right]
$$

where $\eta_{j}^{-}(i)$ and $\eta_{j}^{+}(i)$ are given constants.

A similar strategy can be developed to satisfy this requirement. It is natural to ask if one can choose a value for $F_{I_{j}}(i)\left(j \in \Omega_{2}\right)$ such that both (70) and (81) are satisfied. The answer to this question is positive if the constraints imposed in (70) and (81) do not lead to conflicting choices for $F_{I}(i)$. Otherwise, the answer is negative.

In developing the control strategy, it is assumed that each robot firmly grasps the payload through the contact point. For some advanced applications, flexible grasping may be required. Hence extension of the results to the soft grasp case would be an interesting future research topic. Another issue worth investigating is the effects of load-transitions during "pick-up" and "drop-off" phases.

\section{APPENDIX}

\section{Skew Symmetry of $N_{5}$}

It is sufficient to show that $\mathcal{N}$ is skew-symmetric. In fact since

$$
\omega_{0} \times R=\dot{R},
$$

it follows that

$$
\begin{aligned}
\mathcal{N}= & \frac{d}{d t}\left(T_{0}^{T} R I_{0} R^{T} T_{0}\right)-2 T_{0}^{T} \omega_{0} \times R I_{0} R^{T} T_{0} \\
& -2 T_{0}^{T} R I_{0} R^{T} \dot{T}_{0} \\
= & \left(\dot{T}_{0}^{T} R I_{0} R^{T} T_{0}-T_{0} R I_{0} R^{T} \dot{T}_{0}\right) \\
& +\left(T_{0}^{T} R I_{0} \dot{R}^{T} T_{0}-T_{0}^{T} \dot{R} I_{0} R^{T} T_{0}\right)
\end{aligned}
$$

It can be verified that

$$
\mathcal{N}+\mathcal{N}^{T}=0
$$

which implies the skew-symmetry of $N_{5}$.

\section{ACKNOWLEDGEMENT}

This work was partially supported by the Center for Manufacturing Research at Tennessee Technological University and by NASA grant NAGW-2924.

\section{References}

[1] Alberts, T. E., and D. I. Soloway, "Force Control of a Multi-Arm Robot System," Proc. of the 1988 IEEE Int. Conf. on Robotics and Automation, Philadephia, PA, April 1988, pp. 1490-1496.

[2] Alford, C. O., and S. M. Belyeu, "Coordinated Control of Two Robots Arms," Proc. of the 1984 IEEE Int. Conf. on Robotics and Automation, Atlanta, GA, 1984.

[3] Anderson, J. N., and M. E. Pittelkau, "ReflexAction Position Control and Two-Arm LoadSharing Force Control for Robotic Manipulators," Tech. Report No. MCTR-1-88-1, Center for Manufacturing Research, Tennessee Technological University, January 1988.

[4] Arimoto, S., F. Miyazaki and S. Kawamura, "Cooperative Motion Control of Multiple Robot Arms or Fingers," Proc. of the 1989 IEEE Int. Conf. on Robotics and Automation, Raleigh, NC, March 1987, pp. 1407-1412.

[5] Carignan, C. R., and D. L. Akin, "Optimal Force Distribution for Payload Positioning Using a Planar Dual-Robot," Jour. of Dynamic Systems, Measurement and Control, Vol. 111, 1989, pp. 205-210.

[6] Cole, A., J. Hause and S. Sastry, "Kinematics and Control of Multifingered Hands with Rolling Contact," Proc. of the 1988 IEEE Int. Conference on Robotics and Automation, Philadephia, PA, April 1988, pp. 228-233.

[7] Craig, J. J., P. Hsu and S. Sastry, "Adaptive Control of Robotic Manipulators," Int. Jour of Robotics Research, Vol. 6 (6), 1987.

[8] Furuta, K., K. Kosuge, Y. Shiote and H. Hatano, "Master-Slave Manipulator Based on Internal Model-Following Control Concept," Proc. the 1987 IEEE Int. Conf. on Robotics and Automation, Raleigh, NC, March 1987, pp. 567-572.

[9] Hayati, S., "Position and Force Control of Coordinated Multiple Arms," IEEE Trans. on Aerospace and Electronic Systems, Vol. 24, No. 5, September 1988 , pp. 584-590.

[10] Hsu, P., "Control of Multi-Manipulator Systems - Trajectory Tracking, Load Distribution, Internal Force Control and Decentralized Architecture," Proc. of the 1989 IEEE Int. Conf. on Robotics and Automation, Scottsdale, AZ, May 1989, pp. 1234-1239. 
[11] Hu, Y. R., and A. A. Goldenberg, "An Adaptive Approach to Motion and Force Control of Multiple Coordinated Robot Arms," Proc. of the 1989 IEEE Int. Conf. on Robotics and Automation, Scottsdale, AZ, May 1989, pp. 1091-1096.

[12] Leahy, Jr., M. B., "Compensation of Industrial Manipulator Dynamics in the Presence of Variable Payloads," Int. Jour. of Robotics Research, Vol. 9 (4), 1990, pp. 86-98.

[13] Li, Z., P. Hsu and S. Sastry, "Dynamic Coordination of a Multi-Robot System with Point Contact," Proc. of the 1988 American Control Conference, 1988, pp. 505-510.

[14] Luh, J. Y. S., and Y.-F. Zheng, "Constrained Relations Between Two Coordinated Industrial Robots for Motion Control," Int. Jour. of Robotics Research, Vol. 6 (3), 1987, pp. 60-70.

[15] Mo, L., and M. M. Bayoumi, "Adaptive Control of the Multi-arm Robotic System" Proc. of the $28^{\text {th }}$ IEEE Int. Conf. on Decision and Control, Tampa, FL, December 1989, pp. 1962-1963.

[16] Nakamura, Y., K. Nagai and T. Yoshikawa, "Mechanics of Coordinative Manipulation By Multiple Robotic Mechanisms," Proc. of the 1987 IEEE Int. Conf. on Robotics and Automation, Raleigh, NC, March 1987, pp. 991-998.

[17] Orin, D. E., and S. Y. Oh, "Control of Force Distribution in Robotic Mechanisms Containing Closed Kinematic Chains," ASME Jour. of Dynamic Systems, Measurement, and Control, Vol. 102, 1981, pp. 134-141.

[18] Özgüner, Ü., S. Yurkovich and F. Al-Abbass, "Decentralized Variable Structure Control of a TwoArm Robotic System," Proc. of the 1987 IEEE Int. Conf, on Robotics and Automation, Raleigh, NC, March 1987, pp. 1248-1254.

[19] Pittelkau, M. E., "Adaptive Load-Sharing Force Controller for Two-Arm Manipulators," Proc. of the 1988 IEEE Int. Control on Robotics and Automation, Philadephia, PA, April 1988, pp. 498505 .

[20] Popov, V. M., Hyperstability of Control Systems, Acdemic Press, New York, 1973.

[21] Salisbury, J. K., and J. J. Craig, "Articulated Hands: Force Control and Kinematics Issues," Int. Jour. Robotics and Research, Vol. 1, 1982, pp. 4-17.
[22] Shames, J., Engineering Mechanics: Dynamics, Prentice-Hall, Englewood Cliffs, NJ, 1960.

[23] Song, Y.-D., and J. N. Anderson, "Adaptive Control of a Colleague-Like Multi-Robot System Handling a Common Unknown Object," Proc. of the $30^{\text {th }}$ IEEE Conf. on Decision and Control, Brighton, England, December, 1991.

[24] Song, Y.-D., and J. N. Anderson, "Adaptive Path Tracking Control of Robot Manipulators with Uncertain Payload Dynamics," Systems and Control Letters, Vol. 17, 1991, pp. 59-70.

[25] Song, Y.-D., and J. N. Anderson, "System Stability and Performance Analysis Based on the Generalized System Energy Accumulation: Part I - Criteria Development," Proc. of 1st IEEE Int. Conf. on Control Applications, September, 1992.

[26] Song, Y.-D., and R. H. Middleton, "Dealing with the Impassive Parameter Variation Problem of Robot Manipulators Performing Path Tracking Tasks," IEEE Trans. on Automatic Control, 1992 (to appear).

[27] Spong, M. W., and M. Vidyasagar, Robot Dynamics and Control, Wiley, New York, 1989.

[28] Suh, I. H., and K. G. Shin, "Coordination of Dual Robot Arms Using Kinematic Redundancy," Proc. of the 1988 IEEE Int. Conf. on Robotics and Automation, Philadephia, PA, April 1988, pp. 504-509.

[29] Tarn, T. J., A. K. Bejczy and X. Yun, "Nonlinear Control Algorithms for Multiple Robot Arms," IEEE Trans. On Aerospace and Electronic Systems, Vol. 24, No. 5, 1988, pp. 571-583.

[30] Unseren, M. A., and A. J. Koivo, "Kinematic Relations and Dynamic Modeling for Two Cooperating Manipulators in Assembly," Proc. IEEE Conf. on Systems, Man, and Cybernetics, 1987, pp. 789802.

[31] Walker, I. D., R. A. Freeman and S. I. Marcus, "Analysis of Motion and Internal Loading of Objects Grasped by Multiple Coorperating Manipulators," Int. Jour. of Robotics Research, Vol 10(4), 1991, pp. 396-409.

[32] Walker, I. D., S. I. Marcus and R. A. Freeman, "Distribution of Dynamic Loads for Multiple Cooperating Robot Manipulators," Jour. of Robotics Systems, Vol. 6 (1), 1989, pp. 35-47. 
[33] Walker, M. M., D. Kim and J. Dionise, "Adaptive Coordinated Motion Control of Two Manipualtor Arms," Proc. of the 1989 IEEE Int. Conf. on Robotics and Automation, Scottsdale, AZ, May 1989, pp. 1084-1090.

[34] Yoshikawa, T., and K. Nagai, "Manipulating and Grasping Force in Manipulation by Multifingered Robot Hands," IEEE Trans. Robotics and Automation, Vol. 7, No. 1, 1991, pp. 67-77.

[35] Yun, X., "Nonlinear Feedback Control of Two Manipulators in the Presence of Environmental Constraints," Proc. of the 1989 IEEE Int. Conf. on Robotics and Automation, Scottsdale, AZ, 1989, May 1989, pp. 1252-1259.

[36] Zheng, Y.-F., and J. Y. S. Luh, "Optimal Load Distribution for Two Industrial Robots Handling a Single Object," Proc. of the 1988 IEEE Int. Conf. on Robotics and Automation, Philadephia, PA, April 1988, pp. 344-349. 